\title{
The impact of COVID-19 on patients with hematological malignancies: the mixed-method analysis of an Israeli national survey
}

\author{
Ilana Levy $y^{1,2} \cdot$ Giora Sharf $^{3} \cdot$ Shlomit Norman ${ }^{4} \cdot$ Tamar Tadmor $^{1,2}$
}

Received: 1 December 2020 / Accepted: 28 May 2021 / Published online: 14 June 2021

(c) The Author(s), under exclusive licence to Springer-Verlag GmbH Germany, part of Springer Nature 2021

\begin{abstract}
Background The COVID-19 pandemic required reevaluation of the therapeutic approach and added emotional stress for patients with hematological malignancies at high risk of contracting the virus. We aimed to evaluate how it affected such patients during the second lockdown in Israel.

Methods This national survey included Hebrew-speaking patients with hematological malignancy. This included three tools with 28 items of sociodemographic and medical baseline characteristics, management of hematological disease, and evaluation of emotional coping during COVID-19 pandemic; the Hebrew version of the Patient Health Questionnaire 9; and 3 qualitative open-ended questions. Data was analyzed by mixed methods which combined both quantitative and qualitative thematic analyses.

Results Four hundred eight patients responded to the survey. The management of their hematological disease included a decrease in the number of visits to the hematology clinic (37.0\%), delay of some treatment schedules $(9.1 \%)$, and prescription of replacement therapies permitting less visits to the clinic (2.2\%). The frequency and intensity of "feeling afraid" regarding COVID-19 infection was increased (mean \pm SD: $4 \pm 1$ to $5 \pm 2$ in a 1-7 Likert scale), and a high rate of depression was recorded, which appeared to be more evident in patients with chronic myeloid leukemia (CML) $(p<0.001)$.

Conclusion The management of hematological malignancies during pandemics should always take into consideration patients' fears, as well as the development of depression related to isolation and loneliness, in addition to the high risk of severe disease. Patients with CML had a high rate of depression which obviously needs to be managed very carefully during and after the COVID-19 pandemic.
\end{abstract}

Keywords Hematological malignancies $\cdot$ COVID-19 $\cdot$ Survey $\cdot$ Coping $\cdot$ Depression $\cdot$ Mixed methods

\section{Introduction}

The coronavirus disease 2019 (COVID-19) pandemic first erupted in late 2019 and rapidly spread throughout the world [1]. Patients with comorbidities, including hemato-oncological diseases, have an increased risk of developing serious

Ilana Levy

ilana.levy@b-zion.org.il

1 Hematology Unit, Bnai Zion Medical Center, 47, Golomb Street, 31048 Haifa, Israel

2 The Ruth and Bruce Rappaport Faculty of Medicine, Technion, Haifa, Israel

3 Halil Haor non-profit organization for patients with Leukemia and Lymphoma, Israel, Israel

4 AMEN, Israeli Association of Myeloma Patients, Israel, Israel illness and greater mortality [2,3]. Therefore, in Israel since the beginning of the pandemic, two national population lockdowns have been completed, and patients with chronic illnesses including hemato-oncological malignancies were advised to minimize social activities in order to prevent infection [4]. Optimal approach in terms of patients' visits to the hematology clinic, and decisions regarding changing treatment to mostly oral drugs, was also under investigation worldwide [5]. In addition, emotional aspects received more attention and assumed greater importance during the pandemic in both the general population [6] and infected COVID-19 patients [7]. One should take into consideration that even before the pandemic, depression seemed to be prevalent among patients with hematological malignancy, estimated at $54 \%$ in a Greek study, and associated with decreased quality of life (QOL) and cognitive functioning [8]. Suicide attempts appear to be prevalent among cancer 
patients in general and patients with hematological malignancies in particular [9]. Thus, it is not surprising that during the COVID-19 pandemic, a 36\% rate of anxiety and $31 \%$ rate of depression was recorded among lymphoma outpatients [10]. Coping styles associated with better QOL in cancer patients included emotional support and acceptance, [11] while living alone and insufficient social support seem to be associated with treatment non-adherence in patients with hematological malignancies [12] However in this respect, a recent study showed that patients with chronic conditions such as inflammatory arthritis seem to have a better capacity to successfully cope with stressful events during COVID-19 pandemic [13]. Nevertheless, this point was not truly evaluated among patients with hematological malignancies.

Therefore, the aim of the present study was to evaluate the demographic and clinical factors associated with the emotional conditions of patients with hematological malignancies during the COVID-19 s lockdown in Israel.

\section{Methods}

\section{Study design}

This is a cross-sectional survey conducted among patients with hematological malignancy during the second COVID19 lockdown in Israel. The study was reviewed and approved by the Bnai Zion Medical Center Institutional Review Board in accordance with the Declaration of Helsinki (approval number BNZ-20-0148). Surveys were collected between October 1 and October 21, 2020. They were diffused in Google Forms platform and sent to patients with hematological malignancies via social networks, email, telephone, and nonprofit patient organizations' chat groups (Halil Haor, AMEN). Four reminders to fill in the survey in Hebrew were sent in these platforms during the study period.

\section{Participants}

Hebrew-speaking patients with hematological malignancy were eligible to participate in the study. No informed consent signing was required since the survey was anonymous, and it was made clear to all involved before filling in the questionnaire that the data will be used for statistical analyses and research purposes.

\section{Questionnaires}

The questionnaires were composed of 13 sociodemographic and medical baseline questions, 11 questions on management of hematological disease during COVID-19 pandemic, and 4 questions referring to fears during COVID-19 pandemic in a 1-7 Likert scale. Patients then answered the Patient Health
Questionnaire 9 (PHQ-9) which is a validated tool (translation to Hebrew has been validated as well), assessing the degree of depression as well as the impact of their symptoms on their lives [14]. The total 0-27 PHQ-9 score categorizes depression severity as minimal or none ( $0-4$ score), mild (5-9 score), moderate (10-14 score), moderately severe (15-19 score), or severe (10-27 score). Finally, three open-ended questions about difficulties to be handled, supportive resources, and suggestions to improve the current inconveniences/difficulties were filled by the patients. The analysis of these quantitative and qualitative data was based on mixed methods.

\section{Statistical analysis}

Descriptive statistics were calculated for all demographic and clinical data, including means with standard deviation (SD) and median with ranges (minimum-maximum) for all continuous data, and proportions for discrete and nominal data. For comparing normally distributed variables between more than two study groups, we used the ANOVA test. For comparing discrete and nominal variables, we used the independent Chi-square test and Fisher exact test. Bonferroni adjustment was used for multiple comparisons. Multivariate linear regression analysis was performed after collinearity; interaction and confounding analysis of normally distributed Likert scale survey questions, in order to neutralize the effect of the different sociodemographic and medical covariates. Independent variables included age, sex, hematological disease, time from hematological disease diagnosis, familial status, country of birth, religion, education, occupation, and region of follow-up. The adjusted unstandardized regression coefficients $(\beta)$ were given with confidence interval (CI), while a $p<0.05$ was considered statistically significant. Data analysis was performed using IBM SPSS version 22 statistics software.

\section{Thematic analysis}

Open-ended questions were analyzed using thematic analysis method, which is a variant of qualitative text analysis and examines the occurrence of themes or concepts in texts [15-17]. The analysis was based on open coding procedure, creating categories and abstraction [18] The codes were derived by means of inductive analysis, which is used in cases where no previous studies dealt with the same phenomenon or when such studies are fragmentary [19].

\section{Results}

\section{Baseline characteristics}

Among the total 1206 patients that were approached, 408 answered the survey (response rate 34\%). Among them, 113 
(27.8\%) had chronic myeloid leukemia (CML), 92 (22.7\%) multiple myeloma (MM), 75 (18.5\%) chronic lymphocytic leukemia (CLL), 67 (16.5\%) lymphoma, 41 (10.1\%) had a myeloproliferative neoplasm (MPN), 14 (3.4\%) had acute leukemia, and 4 (1.0\%) hairy cell leukemia. Their baseline characteristics are described in Table 1.

\section{Management of hematological malignancy during COVID-19 pandemic}

Fifty-six (13.9\%) responders did not visit the hematological clinic at any time during the COVID-19 pandemic, while 37 $(9.1 \%)$ had their treatment delayed because of the pandemic. When asking patients about their visits to the hematological clinic since the beginning of the COVID-19 pandemic, 65 $(15.9 \%)$ claimed that their visits had dropped significantly, $86(21.1 \%)$ had their visits slightly decreased, 253 (62.0\%) stated that their visits' frequency was unchanged, and 4 $(1.0 \%)$ had visits slightly increased. Further information on management of hematological malignancies during the pandemic is presented in Table 2 .

\section{Fears during COVID-19 pandemic}

We asked patients to evaluate their fears during the COVID19 pandemic in a 1-7 Likert scale (1: almost never, 7: almost always).

Concerning the statement "I am afraid to contract COVID-19," the mean \pm SD answer was $5 \pm 2$, and the median [minimum-maximum] was 6 [1-7]; and multivariate linear regression analysis showed that women tended to be more afraid than men to contract COVID-19 infection ( $\beta=0.49[0.15 ; 0.83], p=0.005)$.

As for the statement "I am anxious around COVID-19 pandemic in my hematology clinic," the mean answer was $4 \pm 2$, the median was 4 [1-7], and multivariate linear regression analysis showed that women $(\beta=0.58[0.18 ; 0.97]$, $p=0.004)$ and younger people $(\beta=-0.02[-0.04 ;-0.003]$, $p=0.020)$ tended to be more anxious around COVID19 infection in the hematology clinic; every year of age decreased the level of anxiety by 0.02 in the 1-7 Likert scale.

Concerning the statement "The hospital responds to my demands in terms of anxiety around COVID-19 pandemic" (1: not at all, 7: in a significant way), the mean answer was $5 \pm 2$, the median was 6 [1-7], and multivariate linear regression analysis showed that divorced and widowed (as compared to married and single) patients $(\beta=-0.45$ $[-0.77 ;-0.13], p=0.005)$ and patients with longer disease duration $(\beta=0.03$ [0.003; 0.05], $p=0.028)$ felt that the hospital responded better to their demands in terms of anxiety around the pandemic.

Finally, to the statement "If I contract COVID-19, my chances to have serious disease are (1: much lower, 5: much higher) than the general population," the mean answer was $4 \pm 1$, the median was 5 [2-5], and multivariate linear regression analysis showed that women $(\beta=0.22[0.09$; $0.36], p=0.001)$, older patients $(\beta=0.009$ [0.003; 0.02], $p=0.003$ ), and patients with a shorter disease duration $(\beta=-0.011[-0.02 ; 0.00], p=0.040)$ felt that COVID-19 infection may be more serious if they contract it, compared to the general population.

\section{Depression according to PHQ-9 questionnaire}

The mean PHQ-9 score was $9 \pm 6$, and its median was 8 [0-24]. Among the 406 responders to this part of the survey, $91(22.4 \%)$ had no or minimal depression, $143(35.2 \%)$ had mild depression, 100 (24.6\%) had moderate depression, 49 (12.1\%) had moderately severe depression, and 23 (5.7\%) had severe depression (Fig. 1A). When comparing depression categorization between hematological malignancies, it should be noted that CML patients had a significantly more severe degree of depression, with 14 (12.4\%) patients classified as having severe depression $(\mathrm{Chi}-2=22.85, p<0.001)$ (Fig. 1B).

\section{Open-ended questions}

Six categories of burdens/difficulties patients were dealing with during the COVID-19 pandemic were described. When sub-analyzing responses by patients' conditions, those with lymphoma and multiple myeloma mostly had difficulties dealing with isolation and loneliness, while patients with CML, MPN, and CLL mostly described the physical manifestations of their disease and its treatment. Furthermore, ten categories of ways to cope with the different difficulties were described by the 357 responders that stated a difficulty in the preceding open-ended question. Family or friends were underlined by 75 (21.0\%) of them and "being occupied" by $74(20.7 \%)$. Finally, another ten categories of solutions that may have helped improving the abovementioned difficulties were defined by patients. While 98 (27.5\%) could not think of any potential solution, $59(16.5 \%)$ thought of a life without COVID-19 and 57 (16.0\%) believed the restoration of familial and social activities may well improve such difficulties. Quotations and keywords of the thematic analysis are presented in Table 3.

\section{Discussion}

The present study relates to important factors in the life of patients with hematological malignancy during COVID-19 s lockdown in Israel. While the patient management during this period was adapted to the circumstances, responders still disclosed frequent fears, accompanied by a relatively high 
Table 1 Baseline characteristics

\begin{tabular}{l} 
Baseline characteristics \\
\hline Age \\
Mean \pm standard deviation \\
Median [minimum-maximum] \\
Years since diagnosis \\
Mean \pm standard deviation \\
Median [minimum-maximum] \\
Sex \\
Familial status \\
\\
Number of children \\
Mean \pm standard deviation \\
Median [minimum-maximum] \\
I live
\end{tabular}

Religion

Country of birth

Education

Occupation

Hematological disease

Region of hematological follow-up

Treatment for hematological disease
Descriptive

$60 \pm 14$

63 [18-90]

$7 \pm 6$

5 [0-40]

Male - N (\%)

$192(47.1 \%)$

$216(52.9 \%)$

$322(78.9 \%)$

$36(8.8 \%)$

$32(7.8 \%)$

$18(4.4 \%)$

$3 \pm 1$

$3[0-7]$

$356(87.7 \%)$

$48(11.8 \%)$

$2(0.5 \%)$

$396(98.3 \%)$

$7(1.7 \%)$

$289(71.9 \%)$

$42(10.4 \%)$

$27(6.7 \%)$

$23(5.7 \%)$

$12(3.0 \%)$

$9(2.2 \%)$

$279(68.9 \%)$

$28(6.9 \%)$

$66(16.3 \%)$

$32(7.9 \%)$

$140(34.4 \%)$

$121(29.7 \%)$

$69(17.0 \%)$

$32(7.9 \%)$

24 (5.9\%)

$21(5.2 \%)$

$113(27.8 \%)$

67 (16.5\%)

$92(22.7 \%)$

$41(10.1 \%)$

75 (18.5\%)

$14(3.4 \%)$

$4(1.0 \%)$

247 (60.7\%)

$106(26.0 \%)$

$17(4.2 \%)$

$36(8.8 \%)$

$1(0.2 \%)$

$125(30.6 \%)$

$218(53.4 \%)$

101 (24.7\%) 
Table 1 (continued)

Legend: USSR Union of Soviet Socialist Republics

Table 2 Hematological management during COVID-19 pandemic

\begin{tabular}{|c|c|c|}
\hline \multicolumn{2}{|l|}{ Hematological management } & \multirow{2}{*}{$\frac{N(\%)}{249(61.8 \%)}$} \\
\hline During COVID-19 pandemic I come to hematological visits & Personal vehicle or walking & \\
\hline & Public transport & $13(3.2 \%)$ \\
\hline & Taxi & $11(2.7 \%)$ \\
\hline & Accompanied by family & $74(18.4 \%)$ \\
\hline & I do not visit hematological clinic & $56(13.9 \%)$ \\
\hline \multicolumn{2}{|l|}{ I was infected with COVID-19 } & $6(1.5 \%)$ \\
\hline \multicolumn{2}{|l|}{ If yes, I was hospitalized } & $3(50.0 \%)$ \\
\hline \multicolumn{2}{|l|}{ A family member was infected with COVID-19 } & $48(11.8 \%)$ \\
\hline \multicolumn{2}{|l|}{ I was supposed to receive treatment but it was delayed because of COVID-19 pandemic } & $37(9.1 \%)$ \\
\hline \multirow[t]{3}{*}{ If yes, delayed by } & $<1$ week & $1(2.9 \%)$ \\
\hline & 1 week to 1 month & $13(37.1 \%)$ \\
\hline & $>1$ month & $21(60.0 \%)$ \\
\hline \multirow[t]{4}{*}{ Treatment delay was } & In my demand & $8(22.2 \%)$ \\
\hline & Physician decision & $13(36.1 \%)$ \\
\hline & Shared decision & $13(36.1 \%)$ \\
\hline & Other & $2(5.6 \%)$ \\
\hline \multicolumn{2}{|c|}{ I was supposed to have bone marrow transplantation but it was delayed because of COVID-19 pandemic } & $2(0.5 \%)$ \\
\hline \multicolumn{2}{|l|}{ I received an alternative treatment for my hematological disease due to COVID-19 pandemic } & $9(2.2 \%)$ \\
\hline \multirow[t]{3}{*}{ If yes, it was } & In my demand & $1(16.7 \%)$ \\
\hline & Physician decision & $2(33.3 \%)$ \\
\hline & Shared decision & $3(50.0 \%)$ \\
\hline
\end{tabular}

level of depression. The difficulties they experienced during this period differed among the various hematological malignancies, as well as the way they coped with such difficulties and the potential solutions they recommended as help.

\section{How to manage hematological malignancies during COVID-19 pandemic?}

The current survey showed that a relatively large proportion of patients with hematological malignancies did not visit or delayed their visits to the hematology clinic, and some even had their treatments delayed or received alternative treatments instead. Such practices are in accordance with the guidelines for management of hematological malignancies during the pandemic [5]. However, a high level of depression in the survey responders was recorded, and qualitative analysis showed that patients were more frequently worried about isolation or loneliness than about being infected with COVID-19, and that their main advice for improving their coping with such difficulties was restoration of routine social life. In the light of our study results, it seems that management of patients with hematological malignancies should be individualized during pandemics, and that the importance of isolation to prevent contagion should be balanced with the potential psychological and physical consequences of loneliness.

\section{Fears concerning the COVID-19 pandemic}

According to our survey results, women were especially afraid to contract COVID-19, more anxious being around COVID-19 in the hematology clinic, and felt that their chances to contract severe disease if infected with COVID19 were higher. In addition, although older patients justifiably felt that they were more at risk to contract severe disease, they generally felt safe in the hematology clinic around COVID-19 compared to younger patients. Similarly, a recent Italian study showed that outpatients with lymphoma who were at risk of anxiety and post-traumatic stress disorder during the COVID-19 pandemic were mostly women and younger patients [10]. These at-risk populations should be targeted and their needs should be more precisely and individually investigated in order to improve patients' wellbeing in the hematology clinic during such stressful times. 
Fig. 1 Depression assessment by Patient Health Questionnaire 9 (PHQ-9). A Categorization of depression according to PHQ-9 responding. B Categorization of depression in different hematological malignancies. Legend: CML, chronic myeloid leukemia; CLL, chronic lymphocytic leukemia; HCL, hairy cell leukemia; MM, multiple myeloma; MPN, myeloproliferative neoplasm

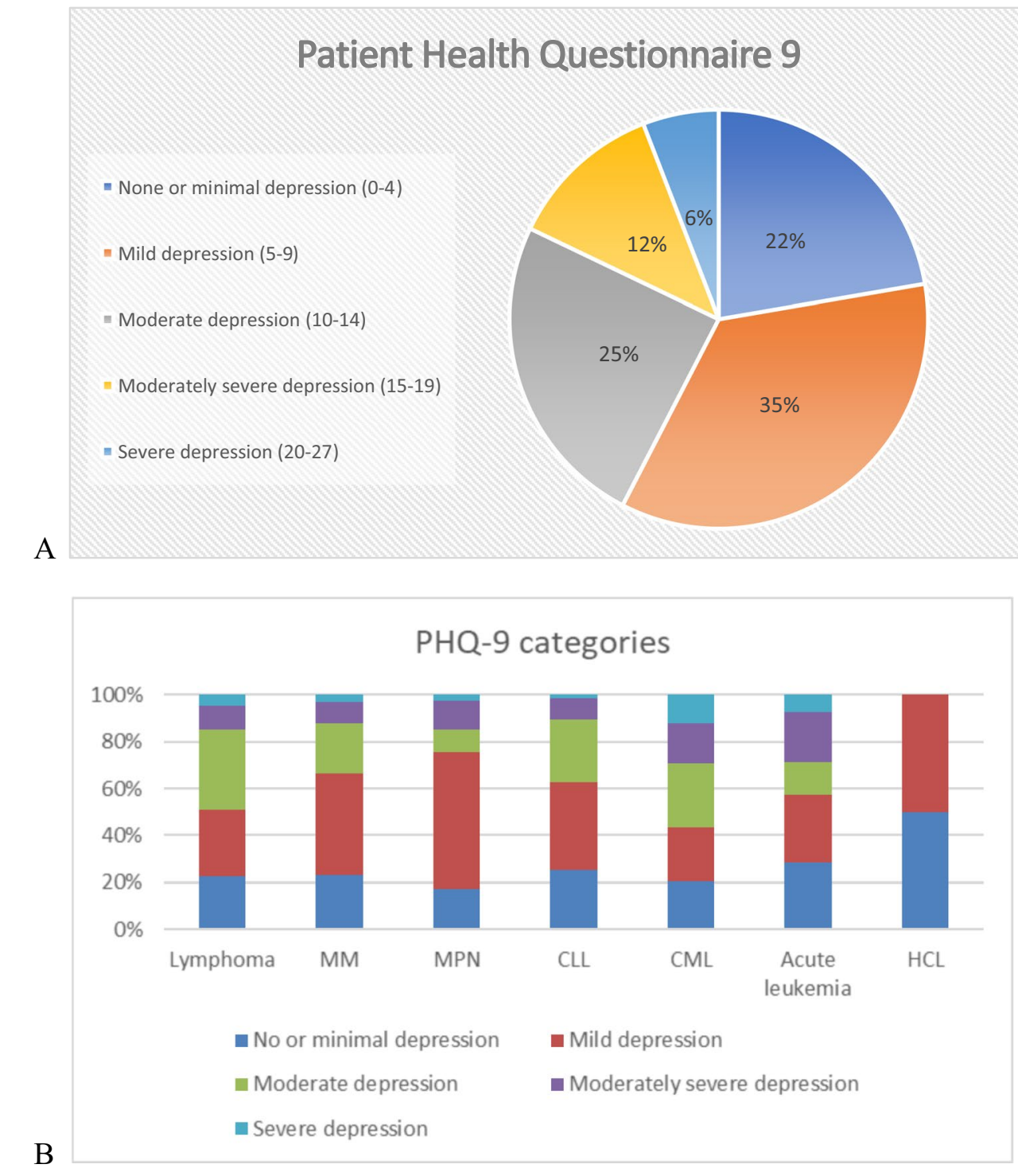

\section{Depression and difficulties during the pandemic}

While a high rate of depression was defined for hematological malignancies, even higher than that recorded among lymphoma outpatients in Italy during the pandemic, [10] it seems that patients with CML were most at risk of severe depression during this period. However, it is still unclear whether this predominance of CML is related specifically to the pandemic. Indeed, thematic analysis showed that these patients displayed apparent COVID-19-unrelated difficulties, essentially physical manifestations of the disease or its treatment. These findings support the fact that the high prevalence of severe depression in patients with CML may not be related to the COVID-19 pandemic or lockdown. An earlier study, published before the COVID-19 pandemic, reinforced our data since both patients and their physicians listed fatigue, muscle cramps, swelling, personal worries, and uncertainty about health condition in the future, as the most important QOL issues in CML [20]. These findings show that despite the pivotal implementation of treatment with tyrosine kinase inhibitors, CML patients still experience major physical and psychological issues related both to the disease itself and its treatment. Future studies should compare the prevalence of depression among patients with CML during and before/after the pandemic. In this respect, it is important that better symptom management and psychological support should be introduced in hematology clinics to help manage these issues in the CML population.

\section{Strengths and limitations}

Our study is a national survey which applied to patients with all types of hematological malignancies, permitting a cost-effective method of collecting data from a large sample 
Table 3 Thematic analysis of open-ended questions

\begin{tabular}{lr}
\hline Themes & Frequency, \\
\hline 1st question: Burdens/difficulties patients were dealing with dur \\
Loneliness and isolation & $113(27.8 \%)$ \\
& \\
Physical manifestations related to the hemato- & $109(26.8 \%)$ \\
logical condition or its treatment &
\end{tabular}
logical condition or its treatment

Fears related to the COVID-19 pandemic

$49(12.1 \%)$

Uncertainty about the future

$31(7.6 \%)$

Fears related to the hematological malignancy

$30(7.4 \%)$

Related to work and economic issues

$19(4.6 \%)$

No difficulty or no answer

$51(12.6 \%)$

2nd question: Main ways to cope with the mentioned difficulties*

Family or friends $\quad 75(21.0 \%)$

Being occupied

$74(20.7 \%)$

No way to cope to difficulties

$37(10.7 \%)$

3rd question: Main solutions that may have helped improving the mentioned difficulties**

Life without COVID-19 $59(16.5 \%)$

Restoration of familial and social activities

$57(16.0 \%)$

$98(27.5 \%)$
Keywords (mentions)

"Friends" (34)

"Family" (23)

"Impossibility to meet family and friends."

"Lockdown" (20)

"Loneliness far from children, family and friends."

"Impossibility to go out, visit family and children, or travel abroad."

"Significant fatigue related to the disease."

"Side effects after each therapy."

"Significant muscle pain and weakness related to treatment with a new drug."

"Loneliness" (7)

"Fatigue" (37)

"Pain" (27)

"Weakness" (14)

"Insomnia" (7)

"Uncertainty about what may happen if I am infected with corona."

"Infected" (39)

"Corona" (23)

"Fear about the pandemic."

"Fear to be infected with corona."

"Fear of the unknown."

"Psychological difficulty and fear of what may be in the future."

"Uncertainty about when it will end."

"Uncertainty and non-understanding about the future of my hematological disease."

"Uncertainty about the efficacy of the treatment I receive."

"Fear that the response to the treatment will not be good enough."

"Uncertainty about my health."

"Uncertainty about the future in an economical and professional point of view."

"I did not come back to work."

"Work" (9)

"Economy" (5)

"Busy" (3)

"Treatment" (11)

"Disease" (8)

"Health" (5)

"Uncertainty" (5)

"The necessity to take care of my children."

"Family" (34)

"Children" (15)

"Family and friends' support."

"Friends" (10)

"The fact that I continue working as usual."

"Distractions such as work, reading, sport..."

"Reading" (19)

"Work" (14)

"I am cooking a lot and painting almost every day."

"Occupy" (6)
"The possibility to go out and meet family normally."

"That corona is temporary."

"Vaccination for corona."

"Going out and meeting friends and family."

"Meeting friends, family, children."

"The opening of swimming-pools and sport areas."
"Lockdown" (18)

"Corona" (13)

"Vaccinate" (9)

"Friends" (17)

"Meeting" (8)

"Family" (7)

*Other ways to cope with the mentioned difficulties: patient's personality (optimism, faith), substance use (drugs or cannabis), technology (e.g. internet, telephone), psychological or complementary medicine treatments, physical activity, rest, protection against COVID-19, hematologist's support

${ }^{* *}$ Other potential solutions: conversations and psychological help, better consideration from the system and the physicians, better health status and normal test results, holidays and vacations, emotional support, substance use (drugs, cannabis), work, physiotherapy 
of patients. Another strength of our survey is its reliability that relates to its standardized diffusion to the participants. Finally, the mixed-method analysis of both quantitative and qualitative data allowed us to approach the study question from different points of view, which facilitates integration of comprehensive data. However, some limitations should also be stressed. Indeed, the generalizability of our study results is limited because they are based only on Hebrew-speaking patients living in Israel, as well as their recruitment by social networks and nonprofit organizations which as mentioned in the results, mainly include Jewish people and patients with basic academic education. Larger international studies should be performed to test the generalizability of our findings. Moreover, the reports of emotional aspects might have been influenced by some data which were not reported in the current study, including number of treatments, relapses/ remission, performance status, etc. Future studies should include these clinical data in order to more reliably examine the factors influencing emotional stress among patients with hematological malignancies. Finally, the recruitment of survey responders via social media may have led to selection bias reflected by the low response rate since these media specifically cater to the younger demographic which may not coincide with the age categories of patients affected by hematological malignancies, and to patients with high socioeconomic level since patients with low per capita income as well as patients with low education have little access to social networks and research.

\section{Conclusion}

The management of hematological malignancies during pandemics can still be improved and should take into account all the high risks of severe disease among immunosuppressed patients, patients' fear relating to the COVID-19 pandemic, and the high level of depression encountered in this population, which seems to be mostly related to individual isolation and loneliness. Unrelated to COVID-19, the care of patients with CML should be improved in terms of symptom management and psychological support.

Authors' contributions All authors contributed in constructing the study design, patient recruitment, article writing and reviewing.

Data and materials availability All data are stored in a repository and can be made available as demand.

\section{Declarations}

Ethics approval The study was approved by the Institutional Review Board of Bnai Zion Medical Center, in accordance with the Declaration of Helsinki.
Consent to participate No informed consent signing was required since the survey was anonymous, and it was made clear to all involved before filing in the questionnaire that the data will be used for statistical analyses and research purposes.

Consent for publication N/A.

Conflicts of interest The authors declare no competing interests.

\section{References}

1. Lake MA (2020) What we know so far: COVID-19 current clinical knowledge and research. Clin Med J R Coll Physicians London 20(2):124-127. https://doi.org/10.7861/clinmed.2019-coron

2. Yigenoglu TN, Ata N, Altuntas F, et al (2020) The outcome of COVID-19 in patients with hematological malignancy. J Med Virol:1-6. https://doi.org/10.1002/jmv.26404

3. Piñana JL, Martino R, García-García I et al (2020) Risk factors and outcome of COVID-19 in patients with hematological malignancies. Exp Hematol Oncol 9(1):1-16. https://doi.org/10.1186/ s40164-020-00177-z

4. Staff T. Ministers agree on full lockdown, more severe than Israel's first. The Times of Israel. https://www.timesofisrael.com/ ministers-said-to-agree-on-full-lockdown-more-severe-than-count rys-first/. Published 2020

5. Terpos E, Engelhardt M, Cook G et al (2020) Management of patients with multiple myeloma in the era of COVID-19 pandemic: a consensus paper from the European Myeloma Network (EMN). Leukemia 34(8):2000-2011. https://doi.org/10.1038/ s41375-020-0876-Z

6. Fu W, Wang C, Zou L et al (2020) Psychological health, sleep quality, and coping styles to stress facing the COVID-19 in Wuhan China. Transl Psychiatry 10(225):1-9. https://doi.org/10.1038/ s41398-020-00913-3

7. Samrah SM, Al-Mistarehi AH, Aleshawi AJ et al (2020) Depression and coping among covid-19-infected individuals after 10 days of mandatory in-hospital quarantine, irbid, jordan. Psychol Res Behav Manag 13:823-830. https://doi.org/10.2147/PRBM. S267459

8. Bellali T, Manomenidis G, Meramveliotaki E, Minasidou E, Galanis P (2020) The impact of anxiety and depression in the quality of life and psychological well-being of Greek hematological cancer patients on chemotherapy. Psychol Heal Med 25(2):201-213. https://doi.org/10.1080/13548506.2019.1695864

9. Zaorsky NG, Zhang Y, Tuanquin L, Bluethmann SM, Park HS, Chinchilli VM (2019) Suicide among cancer patients. Nat Commun 10(1):1-7. https://doi.org/10.1038/s41467-018-08170-1

10. Romito F, Dellino M, Loseto G et al (2020) Psychological distress in outpatients with lymphoma during the COVID-19 pandemic. Front Oncol 10(July):1-6. https://doi.org/10.3389/fonc. 2020. 01270

11. Nipp RD, El-Jawahri A, Fishbein JN et al (2016) The relationship between coping strategies, quality of life, and mood in patients with incurable cancer. Cancer 122(13):2110-2116. https://doi.org/ 10.1002/cncr.30025

12. Bouwman L, Eeltink CM, Visser O, Janssen JJWM, Maaskant JM (2017) Prevalence and associated factors of medication nonadherence in hematological-oncological patients in their home situation. BMC Cancer 17(739):1-8. https://doi.org/10.1186/ s12885-017-3735-1

13. Ciaffi J, Brusi V, Lisi L et al (2020) Living with arthritis: a "training camp" for coping with stressful events? A survey on resilience of arthritis patients following the COVID-19 pandemic. 
Clin Rheumatol 39(11):3163-3170. https://doi.org/10.1007/ s10067-020-05411-x

14. Kroenke K, Spitzer RL, Williams JBW (2001) The PHQ-9: validity of a brief depression severity measure. J Gen Intern Med 16(9):606-613. https://doi.org/10.1046/j.1525-1497.2001.01600 9606.x

15. Boyatzis R (1998) Transforming qualitative information

16. Roberts CW, Roberts BCW (1997) Text analysis for the social sciences: methods for drawing statistical inferences from texts and transcripts

17. Guest G, MacQueen KM, Namey EE (2012) Applied thematic analysis

18. Corbin J, Strauss A (2015) Basics of qualitative research
19. Elo S, Kyngäs $H$ (2008) The qualitative content analysis process. J Adv Nurs 62(1):107-115

20. Efficace F, Breccia M, Saussele S et al (2012) Which healthrelated quality of life aspects are important to patients with chronic myeloid leukemia receiving targeted therapies and to health care professionals? Ann Hematol 91(9):1371-1381. https:// doi.org/10.1007/s00277-012-1458-6

Publisher's note Springer Nature remains neutral with regard to jurisdictional claims in published maps and institutional affiliations. 